\title{
Development of technology for destroying stable water-in-oil emulsions by ultrasonic method at the mobile well production preparing unit
}

\author{
A.V. Lekomtsev, I.B. Stepanenko \\ Mining Faculty, Perm National Research Polytechnic University, Perm, Russia
}

\begin{abstract}
Treatment of production fluids to meet the requirements of the first quality group for commercial purposes is becoming more relevant every year in the Perm Region. Most operational facilities are in the final stages of development and characterized by the high water content of production fluids, which later leads to the formation of water-in-oil emulsions (WOEs) during transportation and field preparation. When treated by traditional methods, such as thermal and thermo chemical gravity sedimentation, stable WOEs are not amenable to destruction. These preparation methods are easy to use but do not always provide the expected result. In this regard, an urgent task is to develop and implement new technologies for the preparation of hydrocarbons, which can be used separately from traditional methods, or in combination with them. This method is called ultrasound impact (USI). This article describes regularities in the influence of various parameters on the efficiency of stable water-in-oil emulsion destruction during field-based treatment with the use of USI. The paper describes the experience of using USI, as well as the high destruction efficiency of stable water-in-oil emulsions in comparison with traditional methods. The authors of the article conducted pilot tests using a mobile unit for treating production fluids with stable WOEs pre-treated by ultrasound. Following test results, the technological effect has been evaluated and recommendations for the practical application of the proposed method have been made.
\end{abstract}

\section{Introduction}

The accumulated experience and fundamental knowledge regarding the processes of emulsion destruction at each of its stages [1] indicate the need to develop technologically applicable solutions with the use of the synergistic effect of ultrasonic and thermo chemical impact in the production fluids treatment. Field-based treatment necessary for rendering production marketable plays one of the key roles within the entire chain of production of hydrocarbon raw materials. Often traditional methods (mechanical, thermo chemical, etc.) do not provide the required result. Searching, developing, and applying new technologies to provide treatment to hydrocarbons is a topical practical task. One of the most promising methods is the ultrasonic impact (USI) used in the destruction of stable water-in-oil emulsions. The mechanism of ultrasonic impact on the water-in-oil emulsions is as follows: ultrasonic waves cause mechanical vibrations in the emulsion and then drive and accelerate 
water droplets (a dispersed phase). This increases the number of collisions of water droplets, their unification, and subsequent sedimentation in oil.

Acoustic treatment of borehole products has been studied by a number of scientific groups, but most of the research has not been implemented in the industry. Different approaches and methods of practical testing of this method are described in Russian and foreign technical literature. It is known that ultrasonic impact in combination with traditional methods of oil treatment allows performing more efficient separation of water and hydrocarbon phases [2-3]. In the survey [4], the effectiveness of the impact is described, and key parameters of ultrasonic exposure affecting the process of destruction of a stable water-in-oil emulsion are identified. In the articles [5-7], factors impacting the efficiency of water-in-oil emulsion separation are identified, and it is noted that a positive synergistic effect of joint application of ultrasonic and chemical exposure has been observed. The basic mechanism of vibration impact on liquid is described in the articles [89], where it was found that high-frequency oscillations of the boundaries of the flattened spheroidal cavity (e.g. a drop of water in oil), whose elastic border is repeatedly deformed, leading to averaging the flow within viscous boundary layers, which in its turn induces a secondary flow within the liquid. In the range of extremely low frequencies, there are no secondary flows relative to the boundary layers, and the averaged vortex flows within the volume of the cavity twist in the opposite direction relative to the high-frequency case. Under ultrasonic impact, these processes will assist in mixing the internal contents of the drop, intermingling the drop's "armor" shell, which may be accompanied by coagulation of closely spaced dispersed units. In the surveys [10-11], an experiment is brought under scrutiny where small-amplitude drop vibrations of two immiscible liquids are studied. The levitation of droplets and acoustic liquid flows within the sound field are represented in the surveys [12-13]. They describe the effect of ultrasonic vibrations per one drop of liquid and show that deformation and distortion of drops occur following the impact of vibrations, and when the intensity of this impact increases, large drops are broken into smaller ones and the stable emulsion is renewed. The mechanisms and effects above are indicative of a high potential of the ultrasound method used for the task in question.

The analysis of the scientific literature has shown that there is no systematic approach to the practical and industrial application of ultrasonic impact on the separation of stable WOEs. It should be noted that the results of well-known studies and their evaluation are often ambiguous and not based on pilot tests at real facilities.

The authors carried out pilot tests of ultrasonic impact on a mobile well production preparing unit for the first time. The results of complex laboratory studies are summarized for further assessment of their applicability and stable emulsion destruction efficiency in the Perm Region, which is the main goal of this study. It was found that acoustic vibrations create favourable conditions for water droplets to merge and that the de-emulsification process is more active than in traditional thermo chemical dewatering.

Every year, up to of 50 thousand $\mathrm{m} 3$ of stable waterin-oil emulsion (WOE) form on the territory of the Perm Region, and it is next to impossible to extract marketable oil from it with existing equipment. Substandard raw materials with a slop oil content of up to $30-40 \%$ cannot be sold on the market of hydrocarbon and oil products, which attaches particular importance to the problem indicated. 


\section{Experiments}

\subsection{Ultrasonic system for emulsion destruction}

As an ultrasonic complex, two piezo-ceramic emitters with installed metal membranes were used. These emitters are capable of sustaining resonant frequency f of 13, 26, $40 \mathrm{kHz}$. The membrane, which is a vibrating shell with a two-way load, distributes and introduces mechanical vibrations into the liquid medium (Figure 1).

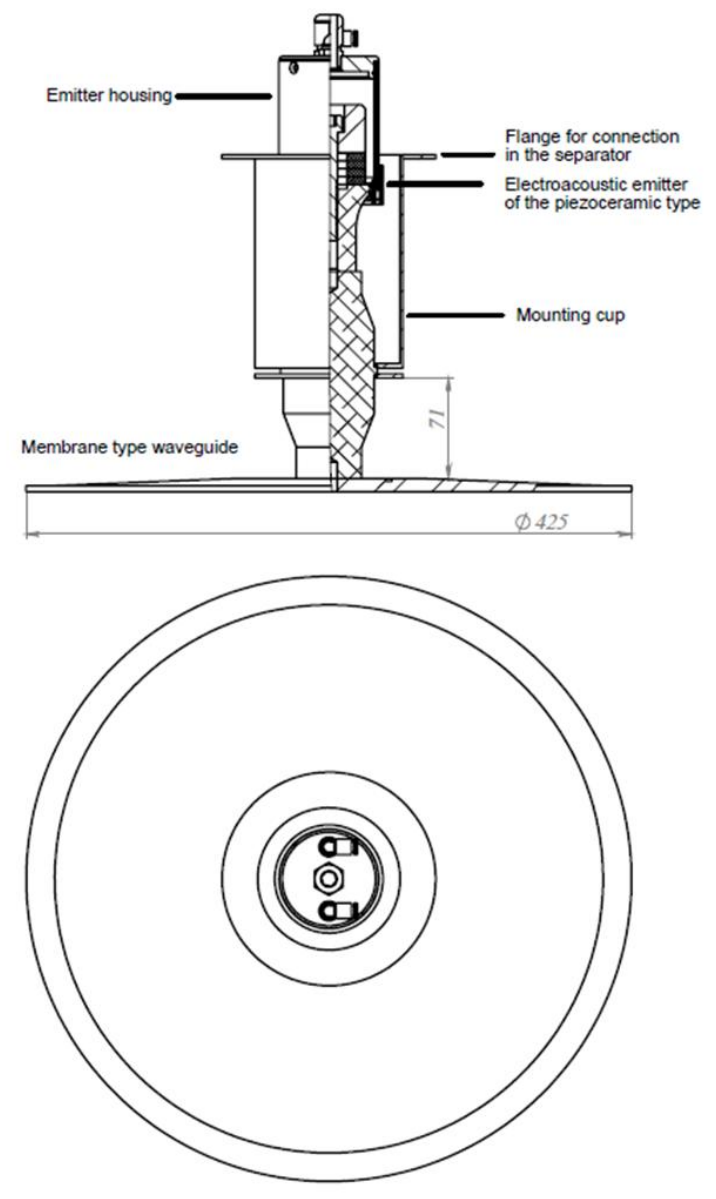

Fig.1.A schematic image of the ultrasonic emitter.

Piezo-ceramic emitters are connected to a single generator to perform synchronous operation. The ultrasonic source is immersed into a vessel with liquid. This scheme is considered to be more flexible in terms of defining the acoustic mode and analyzing its effect on the fluid, and by combining frequencies one may study the impact of ultrasound on the fluid within a wide frequency range. Inside the separator, the waveguide transmits vibrations to a membrane distributing mechanical vibrations over the surface. The flat membrane distributes and transmits ultrasonic vibrations from the source to the liquid within the separator much more evenly than a rod structure. To implement the process of ultrasonic coagulation of liquid-dispersed systems, the treatment shall be carried out in a 
mode close to pre-cavitation, in order to avoid both secondary destruction of water globules just formed and significant dispersion of the internal phase. Maintaining a stable precavitation mode requires a strong restriction of ultrasonic vibrations intensity, which thereby limits the effectiveness of coagulation [14]. In this regard, in order to increase the acoustic energy introduced into the liquid, while preventing cavitation, the USI is carried out in its pulse mode.

The intensity of the pulse impact of the ultrasonic field in the course of the pilot testing (PT) was maintained by a number of cycles during operation and pauses. One cycle of the emitter operation is characterized by a configurable time interval on the impulse generator in terms of width, a number of impulses, and the relaxation period between them (pause time). For convenience, the maximum number of impulses is considered to be 100 , and the minimum is 0 . At a given oscillation power, the relaxation period of a water drop in oil by a stable ultrasonic wave affects the demulsification process significantly. By adjusting the relaxation period, it is possible to establish the best conditions to provide delamination of the emulsion without resorting to artificial cavitation and particle dispersion. To check the significance of the parameters , 3 constant modes of operation of the ultrasonic complex were set with their values in a wide range from $5 / 95$ to $95 / 5$, and the number of cycles in operation/cycles in pause respectively.

\subsection{Physical and chemical properties of water-oil emulsions}

The object of the survey is the intermediate layer within the water preparation tank (WPT), which is a stable reverse WOE. Such an emulsion appears due to the fact that the borderline between water and hydrocarbon phases is vague. Another reason is the accidental pumping of oil-containing liquid imported from the oil field after geological and technical activities have been carried out at the production wells. The quality indicators of the initial water-oil emulsions vary regarding water content from 1 to $85 \%$, and the mass concentration of iron sulfide in the oil phase of the water-oil emulsion differs from 3,900 to 7,900 mg / dm3.

The characteristics of stable water-oil emulsions are shown in Table 1.

Table 1.Physical and chemical properties of stable water-oil emulsion.

\begin{tabular}{llll}
\hline$№$ & Indicator & Units & Value \\
\hline 1 & Oil temperature at $20^{\circ} \mathrm{C}$ & $\mathrm{kg} / \mathrm{m}^{3}$ & $930-970$ \\
2 & Water content (W) & $\mathrm{vol.} \%$ & $1-85$ \\
3 & Salt content $(\mathrm{S})$ & $\mathrm{mg} / \mathrm{dm}^{3}$ & $57000-64000$ \\
4 & Mass fraction of mechanical impurities & $\mathrm{mass} . \%$ & $1,5-1,9$ \\
5 & Mass concentration of iron sulfide in the oil phase & $\mathrm{mg} / \mathrm{dm}^{3}$ & $3900-7900$ \\
6 & WOE viscosity at $20^{\circ} \mathrm{C}$ & $\mathrm{MPa} \cdot \mathrm{s}$ & $150-200$ \\
\hline
\end{tabular}

\subsection{Pilot test}

The process of preparing stable water-oil emulsions at an oil preparation unit (OPU) is performed as follows: the water-oil emulsion is taken from the water preparation tank from the level of 7 metes from below, heated within tubular block furnaces (TBFs) to a temperature of $60 \mathrm{oC}$ and fed into the same WPT to the level of 5 meters from below. 


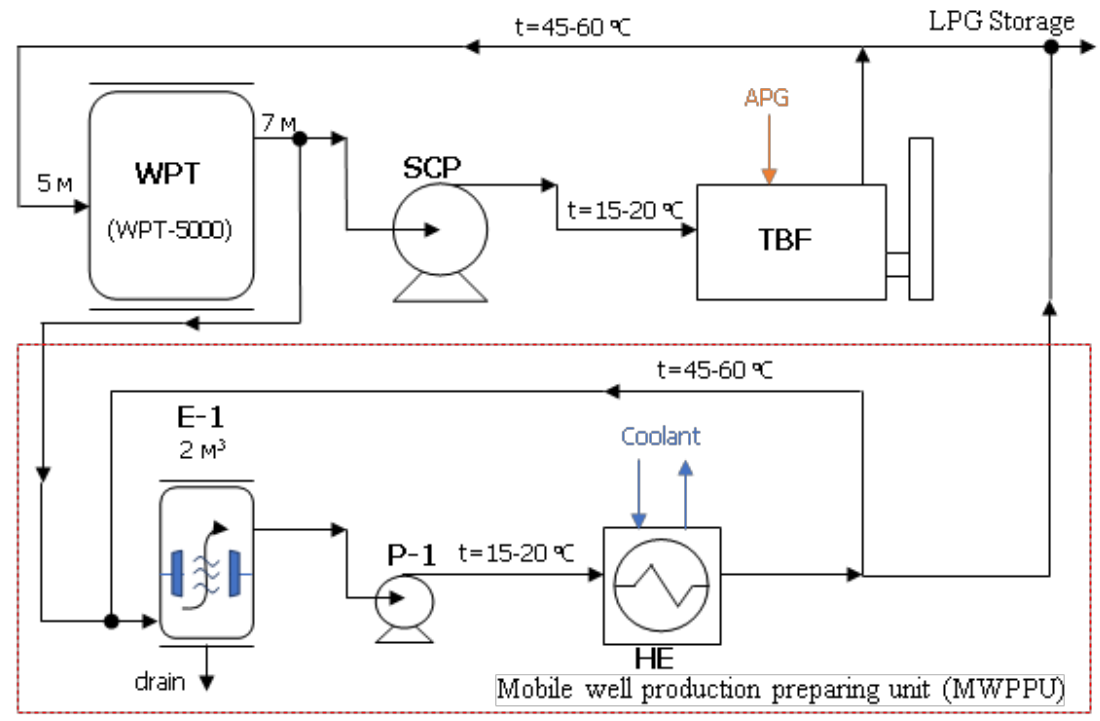

Fig.2.The technological process of preparation of intermediate layers on the OPU with the connection of the mobile well production preparing unit (MWPPU). (HE-heater exchanger; SCP-sectional centrifugal pump; WPT-water preparation tank; P-pump; APG-associated petroleum gas; TBFtubular block furnace).

The preparation of intermediate layers is based on the principle of a heated emulsion recycling with the addition of a demulsifier (DE) FLEC-D028 with its dosage of $200 \mathrm{~g} / \mathrm{t}$ and subsequent gravitational clarification of 12 to 24 hours. This treatment technology allows obtaining oil fluid with its water content of 1.2-1.4\% and average salt content of $1.000 \mathrm{mg} / \mathrm{dm} 3$, which does not meet the requirements of the Russian government requirement GOST R 51858-2002.

Pilot tests of ultrasonic impact technology were performed at a mobile well production preparing unit [15]. One of the functions of the MWPPU is to model new tools and methods for oil and water treatment on the premises of facilities without interfering with or disrupting the main technological process. In order to determine the optimal conditions and destruction modes of stable water-oil emulsions, the MWPPU was connected to the existing Oil Preparation Unit (Perm Region) and operated simultaneously with the flow of the main process (Figure 2). The installation's mode is adjusted in order to accurately simulate the preparation technology on the OPU. Switching into the mode should be implemented as follows: the starting of MWPPU and filling of the E-1 vessel should be performed. Wateroil emulsion with its flow rate of $0.1-0.6 \mathrm{~m} 3 / \mathrm{h}$ at a temperature of $15-20 \mathrm{oC}$ and a pressure of 0.1 MPais fed from E-1 into a closed cycle, heating up to $45-60 \mathrm{oC}$ in a plate heat exchanger until the liquid temperature indicators in the field devices of the OPU match.

The calibration is implemented by adjusting the performance of the MWPPU pump unit. This is based on the WOE preparation period within the devices simulated and the existing technology for preparing well products at the MWPPU being the same, with their thermo-baric conditions identical. After that, the water-oil emulsion is processed by various methods with the quality of the product recorded priorto starting tests within the process and on their completion. The comparison of sample results at the MWPPU input and output is the basis for evaluating the effectiveness of the technology used. 


\section{Results and discussions}

\subsection{The selection of optimal ultrasonic impact modes}

In order to calibrate the MWPPU at the OPU, the raw product brought up from the level of 7 meters from below with its water content of $18 \%$ was heated from 15 to $60 \mathrm{oC}$, with the treatment mode being that of "hot" gravitational sedimentation. According to the results of the procedure, it turned out to be impossible to prepare the water-oil emulsion to conform to the requirements of GOST R 51858-2002, the product's water content is $1.4 \%$, and the content of chloride salts $925 \mathrm{mg} / \mathrm{dm} 3$. The results regarding the quality of the product prepared at the MWPPU using this technology are comparable to the deliverables of laboratory analyses of the water-oil emulsion within the OPU water preparation tank, which is indicative of the high accuracy of the process modeling at the MWPPU.

Next, to exclude the heating effect the optimal intensity of USI was selected at a temperature of $30{ }^{\circ} \mathrm{C}$. The ultrasonic treatment was carried out for three hours at a frequency of 13-14 kHz. This was followed by gravitational sedimentation for three hours. The figure below depicts the result of evaluating the ultrasonic treatment technology effectiveness depending on the impact intensity.

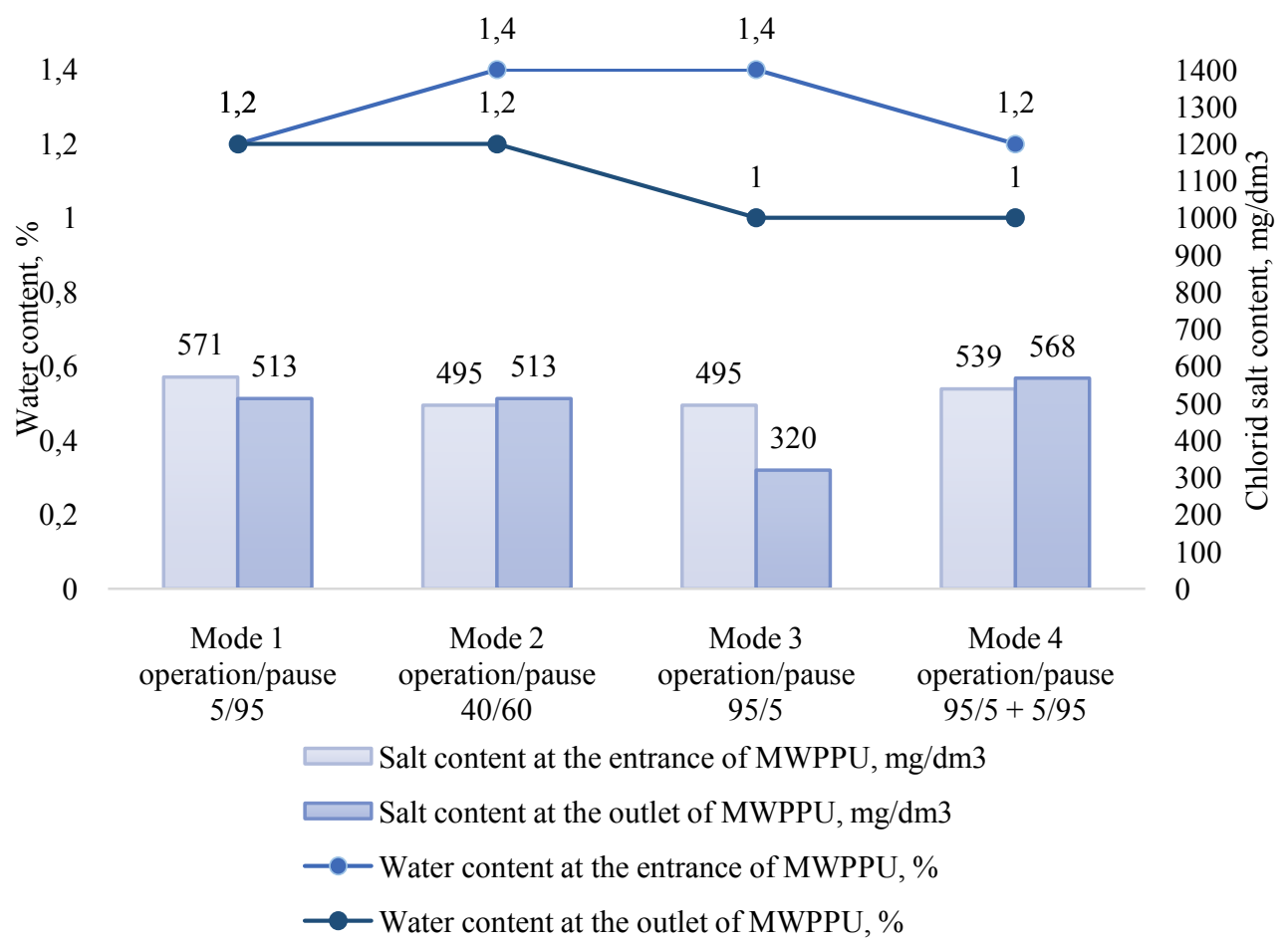

Fig.3.The results of samples analysis past 7 hours of dynamic sedimentation under various modes of the USI operation.

It was found that the best quality indicators were achieved within mode 3 (operationpause 95/5) : the reduction of water content from 1.4 to $1.0 \%$ and the volume of chloride salts - from $495 \mathrm{mg} / \mathrm{dm} 3$ to $320 \mathrm{mg} / \mathrm{dm} 3$ at the MWPPU output, which corresponds to quality group 3 . Within operation mode 4 , the combined impact of the ultrasonic field along with the "operation-pause" parameter - 95/5 (stage 1 - intensification) and then 5/95 (stage 2 - stabilization) was tested. The figure shows that this approach does not worsen the water 
content indicators achieved at the first stage of ultrasonic treatment, but at stage 2 there is a slight increase in salt content after gravitational sedimentation. The reduction of water content in oil fluid below $1 \%$ does not occur due to the high viscosity of the emulsion, which is heated up to the temperature of $30 \mathrm{oC}$. The degree of readiness of raw materials with their initial water content of $1.2-1.4 \%$ is quite low. This is due to the small amplitude of water droplet vibrations compared to the distance between the globules of the dispersed phase. The USI effect may be enhanced by reducing the viscosity of the dispersion medium (oil) by its heating. Further experiments and technological processes are recommended to be carried out at a temperature of at least $50 \mathrm{oC}$.

In order to assess temperature influence on the efficiency of the destruction process of stable water-oil emulsions, a series of experiments were conducted, and their parameters are shown in Table 2.

Table 2.The characteristics of WOE destruction modes 4, 5, 6 under different temperatures.

\begin{tabular}{lllllll}
\hline \multirow{2}{*}{$\begin{array}{l}\text { Mode } \\
\text { number }\end{array}$} & \multicolumn{2}{l}{ Quality of incoming WOE } & \multicolumn{2}{l}{ Quality of output WOE } & $\mathrm{t},{ }^{\circ} \mathrm{C}$ & $\mathrm{f}, \mathrm{kHz}$ \\
\cline { 2 - 7 } & $\mathrm{W}, \%$ & $\mathrm{~S}, \mathrm{mg} / \mathrm{dm}^{3}$ & $\mathrm{~W}, \%$ & $\mathrm{~S}, \mathrm{mg} / \mathrm{dm}^{3}$ & & \\
\hline 4 & 1,2 & 539 & 1,0 & 568 & 30 & 14 \\
5 & 4,0 & 2580 & 0,8 & 629 & 50 & 14 \\
6 & 2,5 & 1048 & 0,9 & 240 & 60 & 14 \\
\hline
\end{tabular}

Figure 4 displays a histogramme that characterizes the effectiveness of ultrasonic application under different temperatures of water-oil emulsion in terms of the content of water and chloride salts within the oil fluid.

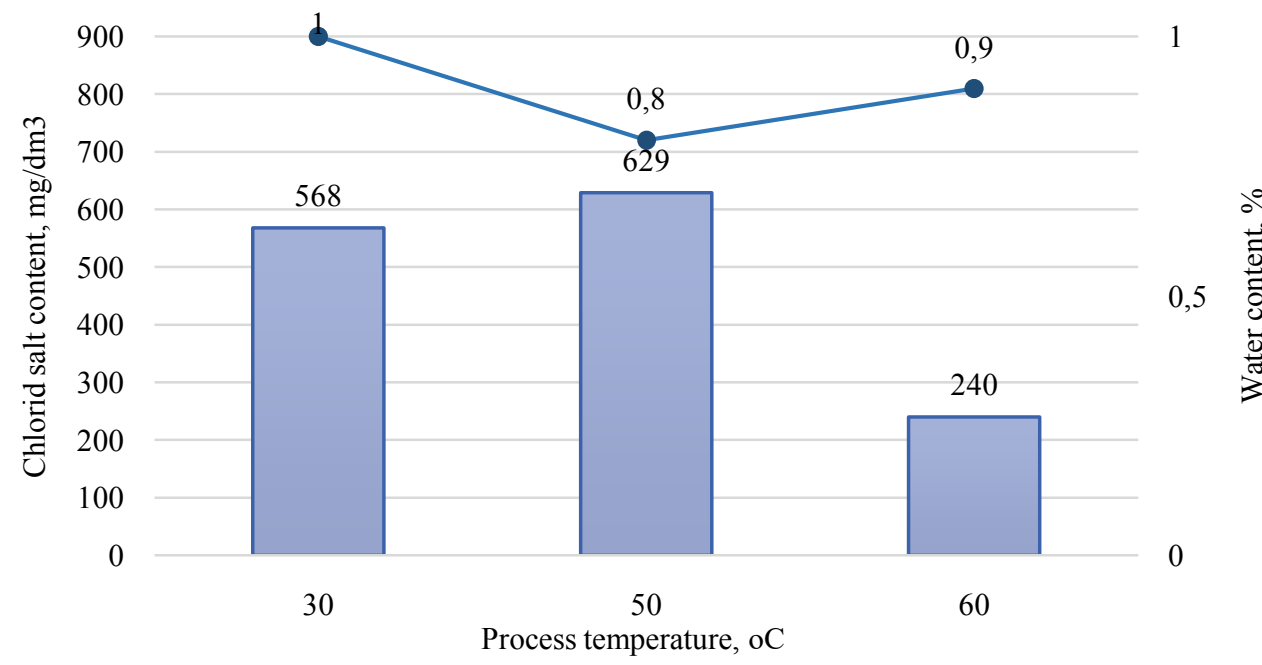

$\square$ Salt content at the outlet of MWPPU, mg/dm3

- Water content at the outlet of MWPPU, \%

Fig.4.The destruction results of stable water-oil emulsions in different temperatures.

In the course of the experiments, it was found that on the emulsion's temperature reaching $60^{\circ} \mathrm{C}$ the optimal efficiency of the technological process is achieved. As the temperature increases, the boundary tension, as well as the viscosity, decreases significantly. There is a decrease in boundary tension in boundary instability, which increases the number of dispersed phase drops. As the temperature increases, the number of nuclei leading to insignificant cavitation within the boundary zone close to the membrane 
plates may grow due to the increase in the vapor pressure of the cavitation medium. With the cavitation events growing and intensity increasing, the deformation of large droplets along with the formation of small ones is observed. Thus, thepat process of water-oil emulsion renewal is most effective, and due to the decrease in viscosity, the coalescence process runs faster under the USI stabilization mode. In addition, with water content in feedstock increased, dewatering occurs more intensively (mode 5), which is accounted for by the improvement in the USI conditions due to the magnified dimensions of the dispersed phase and a shorter distance between water globules. A detailed survey of the dispersion composition allows identifying main regularities of the course of the WOE destruction mechanism.

\subsection{Dispersion analysis of stable water-oil emulsions}

The collected samples of a stable WOE in mode 5 were examined within the scope of laboratory tests in order to determine sample dispersion, to assess the possibility of settlement during gravitational sedimentation, and to compare them with the results of pilot tests. Micro-photos of the samples were taken using MBS-10 microscope with its ToupCam video eyepiece.

Table 3.The results of dispersion analysis of samples before and after the USI (Mode 5).

\begin{tabular}{lcc}
\hline Parameter & \multicolumn{2}{c}{ Sample } \\
\cline { 2 - 3 } & Before the impact & After the impact \\
\hline Water content, \% & 4.0 & 0.8 \\
Average size of water droplets & 7.74 & 5.41 \\
in oil, microns & & \\
\hline
\end{tabular}

Figure 5 shows micro-photographs of samples: on the left is a sample of the initial emulsion from the WPT, on the right it has been USI processed, heated to $50 \mathrm{oC}$, and fed with a demulsifier with its dosage of $50 \mathrm{~g} / \mathrm{t}$. Gravity settlement duration - 12 hours.

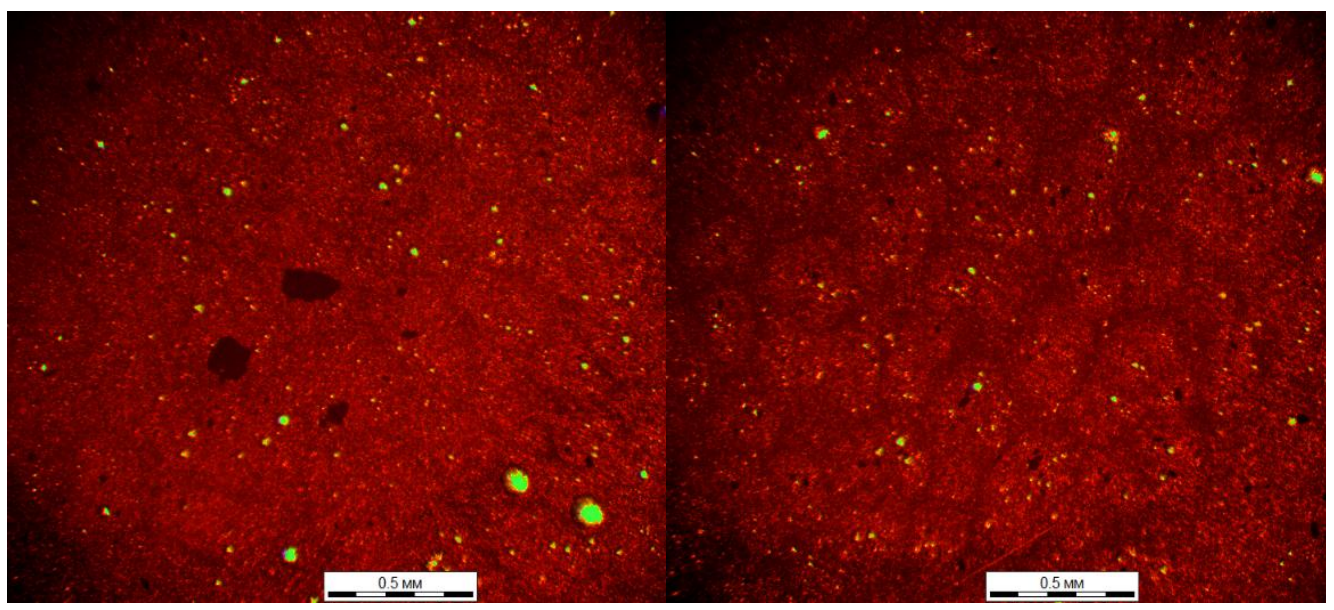

Fig.5.Micro-photos of water-oil emulsion samples before and after USI.

Based on the micro-photos obtained, the structure of the water-oil emulsion was analyzed and primary statistical processing was conducted regarding the distribution of 
water globules in terms of dimensions. The results are shown in Figure 6. The sample reveals solid particulate matter with diameters measuring 7 to 205 microns.

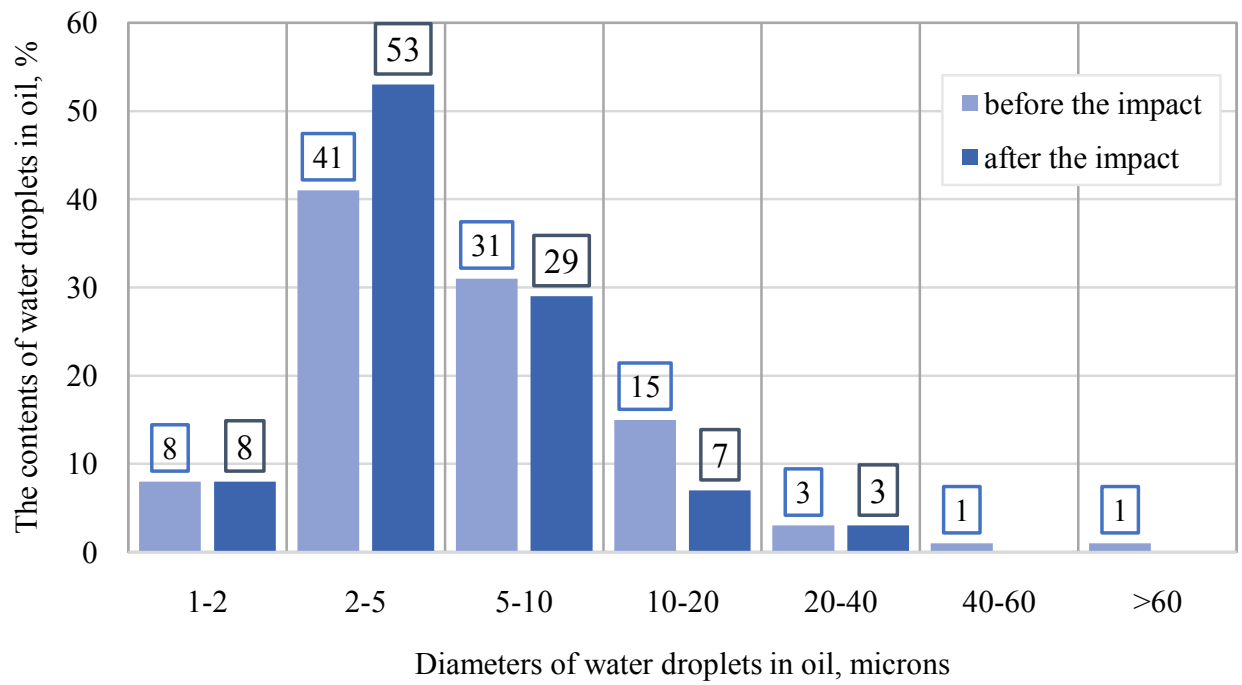

Fig.6.The distribution of the dispersed phase in samples before and after USI (Mode 5).

It was found that the average size of water globules within the initial water-oil emulsion from the WPT is 7.74 microns, and within the processed sample after ultrasonic impact, this value is 5.41 microns. After the complex ultrasonic and thermo chemical treatment, the concentration of water globules measuring upwards of 5 microns decreased. There was a coalescence of droplets, accompanied by a decrease in water content from $4 \%$ to $0.8 \%$. The average dimensions of water globules within the sample decreased by $30 \%$. This analysis confirms the fact that the mechanism of ultrasound action does affect the WOS. Ultrasonic vibrations drive and accelerate water droplets, which contributes to an increase in the number of their collisions, with their further blending and sedimentation in oil.

\subsection{Determination of sedimentation time of water globules in oil}

The sedimentation time of water globules in oil and the operating range of droplets involved in the process of WOE destruction was estimated using the Stokes formula:

$$
\vartheta w=\frac{d_{w}^{2}\left(\mathrm{p}_{w}-\mathrm{p}_{o}\right) g}{18 \mu_{o}}
$$

where $\vartheta_{\mathrm{w}}$ is the sedimentation rate of water globules in oil; $\rho_{\mathrm{w}}$ is the density of bedding water $\left(\rho_{\mathrm{w}}=1135 \mathrm{~kg} / \mathrm{m}^{3}\right) ; \rho_{\mathrm{o}}$ is the density of oil $\left(\rho_{\mathrm{o}}=886 \mathrm{~kg} / \mathrm{m}^{3}\right) ; d_{\mathrm{w}}$ is the diametre of water globules; $\mu_{\mathrm{o}}$ is the dynamic viscosity of bedding oil $\left(\mu_{\mathrm{o}}=10 \mathrm{mPa} \square \mathrm{s}\right)$. The characteristic values are specified for the temperature of $50{ }^{\circ} \mathrm{C}$.

The proportion of water within the volume of drops of the corresponding diametre was determined as

$$
W_{i}=\frac{d_{i}^{3} C_{i}}{\sum d_{i}^{3} C_{i}}
$$

The simulation results are shown in Table 4. 
Table 4.The results of modeling the process of globules sedimentation in the feedstock (Mode 5).

\begin{tabular}{cccccc}
\hline $\begin{array}{c}\text { Diameter } \\
\left(\mathrm{d}_{\mathrm{i}}\right), \\
\text { microns }\end{array}$ & $\begin{array}{c}\text { The content } \\
\text { of the water } \\
\text { globules }(\mathrm{Ci}), \\
\%\end{array}$ & $\begin{array}{c}\text { Deposition } \\
\text { rate, } \\
\text { microns/s }\end{array}$ & $\begin{array}{c}\text { Gravitational } \\
\text { sedimentation }\end{array}$ & $\begin{array}{c}\text { The proportion of water in } \\
\text { the volume of drops of the } \\
\text { corresponding diameter } \\
\text { Wi, \% }\end{array}$ \\
\cline { 4 - 5 } & 8 & 0.05 & 0.25 & 0.5 & \\
\hline 2 & 41 & 0.34 & 1.45 & 2.9 & 0.01 \\
5 & 31 & 1.36 & 5.85 & 11.7 & 0.66 \\
10 & 15 & 5.43 & 23.45 & 46.9 & 3.97 \\
20 & 3 & 21.7 & 93.80 & 187.6 & 24.38 \\
40 & 2 & 48.9 & 211.05 & 422.1 & 55.37 \\
60 & 2 & & & \\
\hline
\end{tabular}

If there are particles with a diameter of 40 or more microns involved in the process of coalescence and gravitational sedimentation in the conditions listed above, their volume fraction of $\mathrm{W}_{40+}$ will be $79.98 \%$. This corresponds to the fraction of bound water in the water-oil emulsion. This water has enough time to sedimentation after the treatment. In practice, the result was a decrease in the water fraction by $80 \%$. Thus, in field conditions, the impact on the particles with their dimensions from 40 microns or above involved in the process of gravitational sedimentation in tanks and devices when operating in the "accumulation - hot settling" mode for at least 12 hours, is considered to be significant.

\subsection{Evidence of ultrasonic impact effectiveness}

The evaluation of the effectiveness of ultrasonic impact was made in the course a series of experiments with different initial water content in water-in-oil emulsion. The characteristics of the WOE preparation modes surveyed are shown in Table 5.

Table 5.The characteristics of modes 6-10.

\begin{tabular}{|c|c|c|c|c|c|c|c|}
\hline \multirow{2}{*}{$\begin{array}{l}\text { Mode } \\
\text { number }\end{array}$} & \multirow[t]{2}{*}{$\mathrm{t}, \mathrm{oC}$} & \multirow[t]{2}{*}{$\mathrm{f}, \mathrm{kHz}$} & \multirow{2}{*}{$\begin{array}{c}\mathrm{DE}, \\
\mathrm{g} / \mathrm{t}\end{array}$} & \multicolumn{2}{|c|}{ Quality of incoming WOE } & \multicolumn{2}{|c|}{ Quality of output WOE } \\
\hline & & & & $\mathrm{W}, \%$ & $\mathrm{~S}, \mathrm{mg} / \mathrm{dm} 3$ & $\mathrm{~W}, \%$ & $\begin{array}{c}\mathrm{S}, \\
\mathrm{mg} / \mathrm{dm} 3\end{array}$ \\
\hline 7 & 60 & 14 & - & 85 & 5100 & 0.9 & 559 \\
\hline 8 & 58 & 14 & 50 & 45 & 4000 & 0.5 & 287 \\
\hline 9 & 50 & 14 & 50 & 4 & 2580 & 0.8 & 629 \\
\hline 6 & 58 & 14 & 50 & 2.5 & 1048 & 0.9 & 240 \\
\hline 10 & 59 & 22 & 50 & 1 & 678 & 0.8 & 454 \\
\hline
\end{tabular}

In the course of the survey, it was determined that in order to increase the efficiency of demulsification of stable water-oil emulsions a demulsifier reagent is crucial. The optimal dosage of the demulsifier reagent in combination with ultrasonic impact is set at $50 \mathrm{~g} / \mathrm{t}$. Further increase in dosage does not improve the result. The optimum dosage $(50 \mathrm{~g} / \mathrm{t})$ is significantly lower than that used in traditional technologies of field treatment of stable water-oil emulsions $(200 \mathrm{~g} / \mathrm{t})$. This is so due to better dispersion of the demulsifier within the water-oil emulsion on USI. It also confirms the applicability of the results of studies in the field of methods concerning the addition, input, and "delivery" of dispersed phase to the border of globules, with the dispersed phase distributed within the dispersion medium [1617].

A comparative analysis of the effectiveness of ultrasonic impact on various water-in-oil emulsions is shown in Figure 6. All experiments on ultrasonic impact were carried out using combined exposure to the ultrasonic field with its variable parameter "operation- 
pause" - 95/5 (stage 1 - intensification) and then $95 / 5$ in the impulse mode (stage 2 stabilization).

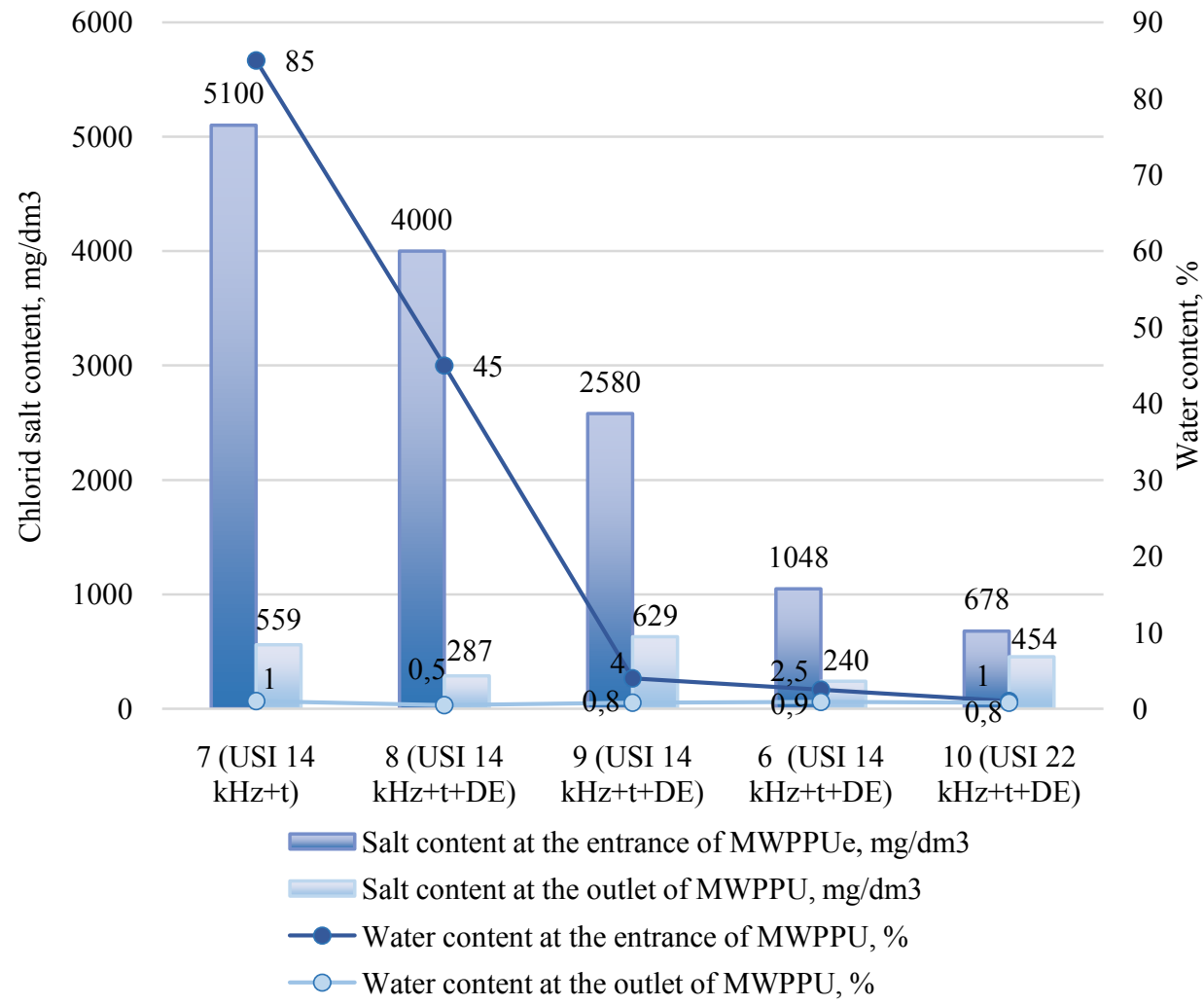

Fig.7.The destruction results of stable water-oil emulsions with different initial water content.

It has been found that the effectiveness of ultrasonic impact is reduced significantly if the water content is below 4\%. Raw materials with the water content of $2.5 \%$ (mode 6 ) and $1.0 \%$ (mode 10) are dewatered by 64 and $20 \%$ respectively. The water-oil emulsion with the water content of $85 \%$ (mode 7 ) and $45 \%$ (mode 8 ) is $99 \%$ dewatered in both cases.

\section{Conclusion}

1) Ultrasonic impact on stable water-oil emulsions with a low water content of $4 \%$ and lower in a mode close to cavitation, renews the emulsion. This encourages new water drops to form, but in the stabilization mode, the oscillation amplitude of some drops is smaller than the distance between them. Thus, not all globules of the dispersion phase coalesce in the course of the settling process. An equally important reason that decreases USI effectiveness is that in the course of ultrasonic treatment the threshold prevailing concentration of water droplets is reached, while vibrations from the emitter are damped significantly. Due to a large distance between the oscillating water droplets, the probability of collision and enlargement of the droplets decreases, which means that the demulsification process either stops or continues very slowly. To completely separate water-oil emulsions, it is required that the amount of treatment time in the stabilization mode be rather long. This allows us to conclude that the use of ultrasonic impact is least effective with emulsions whose water content is below $4 \%$ and above $90 \%$. For effective 
application of ultrasonic technology of stable water-oil emulsions destruction, it is recommended to process feedstock within this specified limited range of water content.

2) The initiating effect of an ultrasonic impact is associated with an increase in the dispersion of the system and, as a result, with the area of contacting surfaces of water globules getting larger. This increases the probability of droplet coalescence of the dispersed phase. Also, as ultrasound waves are generated and the distance between globules grows, the conditions of delivering the demulsifier reagent to the surface of globules are improved, which is favorable for stimulating the demulsification process.

3) Lower feed rates of the demulsifier yield positive results. In the course of the pilot tests (PT), it was possible to justify the reduction in the dosage from 200 to $50 \mathrm{~g} / \mathrm{t}$.

4) For treatment in field conditions, it is the ultrasonic impact on low-water oil in which globules with diametres measuring 40 microns or bigger are exposed to the significant treatment. It is also possible to reduce the proportion of bound water to $80 \%$ in the course of gravitational sedimentation within tanks and devices when operating in the "accumulation - hot sedimentation" mode for at least 12 hours.

Under conditions of pilot testings in a mobile well production preparing unit, a conclusive confirmation of the synergistic effect of complex ultrasonic and thermo chemical impacts on the destruction of stable water-in-oil emulsions was obtained.

\section{References}

1. I.I.Mazein, A.V.Usenkov, A. Yu.Durbazhev, A.V.Lekomtsev, P.Yu. Ilyushin.Fieldtest of coalescer in the mobile unit for well production treatment. Oil economy. 6: 136139.(2018)

2. E.S.Afanasiev, B.I.Rimarenko, Yu.P.Yasyan.. Influence of ultrasonic action on the process of destruction of water-oil emulsions. Oil refining and petrochemistry. 9: 3941.(2009)

3. YeGuoxiang, LuXiaoping, HanPingfang. Desalting and dewatering of crude oil in the ultrasonic standing wave field. Petroleum Science and Engineering. 70: 40-144.(2010)

4. A.V.Lekomtsev, P.YuIlyushin, K.A.Derendyaev, I.BStepanenko, V.A.Mordvinov, R.V. Dvoretskas. Destruction stable water-oil emulsion with the use of ultrasonic impact. Chemical and oil and gas engineering. 2: 9-12.(2019)

5. X.-g.Yang, W. \& X.-f. Tan. Demulsification of Crude Oil emulation via Ultrasonic Chemical Method. Petroleum Science and Technology. 27: 2010-2020.(2017)

6. B.P. Singh,B.P.Pandey.Ultrasonication for breaking water-in-oil emulsions. Proc. Indian natn. Sci. Acad. 3: 181-194.(1992)

7. M.Amani, M. Idris, M.Abdul Ghani, N.Dela Rosa, A. Carvero..An Experimental Study on the Application of Ultrasonic Technology for Demulsifying Crude Oil and Water Emulsions. Journal of Petroleum and Environmental Biotechnology.7: 1-12.(2017)

8. V.G.Kozlov, R.R. Sabirov, S.V. Subbotin.. Averaged flows in an oscillating spheroidal cavity with an elastic wall. Proceedings of the Russian Academy of Sciences of fluid and gas mechanics. 2: 16-26.(2018)

9. A.A.Ivanova, V.G. Kozlov.. Vibrational convection in non-accessible cavity vibrations isothermal case). IzvestiyaRossiyskoyAkademiiNauk. Fluid and gas mechanics. 2: 2532.(2003)

10. C.L.Shen, W.J.Xie, Z.L. Yan, B. Wei. The internal flow of acoustically levitated drops underground sectorial oscillations. Physics Letters A. 374: 4045-4048.(2010) 
11. E., Trinh, A. Zvern, T. G. Wang. An experimental study of small-amplitude drop oscillations in immiscible liquid systems. Journal of Fluid Mechanics. 115: 453474.(1982)

12. V. Niborg. Acoustic currents. Physical acoustics. 1969: 302-377.(1969)

13. D.Zang,Y. Yu, Z.Chen, X.Li, H.Wu, X. Geng. Acoustic levitation of liquid drops: Dynamics, manipulation, and phase transitions. Advances Colloid Interface Science. 243: 77-85.(2017)

14. V.N. Khmelev, R.N. Golykh, M.V.Khmelev, V.A.Shakura, A.V.Shalunov, R.V. Barsukov. Identification of optimal modes of ultrasonic pulse action for coagulation in liquid-dispersed media. South-Siberian Scientific Bulletin. 3: 15-20.(2017)

15. Installation of preparation of well products: Pat. ROS. Federation, no. 2016114262, declared 2016.04.12; publ. 2017.04.17.

16. A.V. Dengaev, A.,A.Getalov, V.S.Verbitsky,M.M. Farkhutdinov. Application of acoustic methods of separation of water-oil emulsions. Report at the international scientific and technical conference Geopetrol: 17-20.(2018)

17. A.V. Dengaev, V.S.Verbitsky, I.T. Mishchenko, A.A. Getalov. Prospects for the use of ultrasonic influence in the process of oil preparation on. Oil economy. 4: 28-30.(2020) 